\title{
On the quantization of a self-dual integrable system
}

\author{
Alex Kasman \\ Department of Mathematics, College of Charleston, 66 George Street, Charleston, SC 29464 USA
}

Received 14 May 2001

Published 3 August 2001

Online at stacks.iop.org/JPhysA/34/6307

\begin{abstract}
In this note, we apply canonical quantization to the self-dual particle system describing the motion of poles to a higher rank solution of the KP hierarchy, explicitly determining both the quantum Hamiltonian and the wavefunction. It is verified that the quantum Hamiltonian is trivially bispectral (that is, that the wavefunction can be taken to be symmetric) as predicted by a widely held hypothesis of mathematical physics.
\end{abstract}

PACS numbers: 02.30.Ik, 45.20.Jj, 45.50.-j

\section{Introduction}

Following Ruijsenaars [14], it has been recognized as convenient and useful to classify integrable particle systems into dual pairs. Roughly speaking, one system is dual to another when the linearizing map of one system is the inverse of the linearizing map of the other. More specifically, a system is said to be self-dual if it is linearized by an involution. (The Calogero-Moser particle system is the best known self-dual integrable system [1].) Duality of integrable systems has been the focus of much recent research due to its role in theories of quantum gravity (see, e.g., $[2,12]$ ). In particular, one observation is that the quantized version of these systems should demonstrate a symmetry of spatial and spectral parameters in their wavefunctions [5,7]. Since this is a special instance of the bispectral property [6], this conjecture will be referred to here as the bispectral quantization hypothesis $(\mathrm{BQH})$.

Although there is no particular reason to disbelieve the $\mathrm{BQH}$, it is not supported by a mathematical proof. (In fact, the ambiguities of the procedure known as 'quantization' would make it difficult to state the $\mathrm{BQH}$ in a verifiable manner.) At present, at least, it is supported only 'experimentally' by the fact that it happens in all the well known systems (Calogero-Moser, Ruijsenaars-Schneider, Sutherland, etc) and by the fact that it 'seems like the appropriate analogue'. It is therefore of interest, when confronted with a new example of a pair of integrable systems related by classical duality, to determine whether it might provide a counter-example. The purpose of this paper is to confirm that the quantum Hamiltonian

$$
\tilde{\mathcal{H}}=\left(\sum_{i=1}^{n} \partial_{i}^{2}-x_{i}\right)-\left(\sum_{1 \leqslant i<j \leqslant n} \frac{4}{\left(x_{i}-x_{j}\right)^{2}}\right)
$$


(corresponding to a self-dual classical system first explicitly studied in [13]) does have a wavefunction with the predicted symmetry. In addition, this result is of interest since it provides new examples of bispectral commutative rings of partial differential operators containing Schrödinger operators and demonstrates that the operator $\tilde{\mathcal{H}}$ intertwines with the operator $\mathcal{H}=\sum_{i=1}^{n} \partial_{i}^{2}-x_{i}$ (in contrast to the case $n=1$ where $\mathcal{H}$ is known not to intertwine with any other rational operators).

\section{Classical particle dynamics}

In this section of the paper we will be concerned with the $n$-particle dynamical system determined by the Hamiltonian

$$
H=\left(\sum_{i=1}^{n} y_{i}^{2}-x_{i}\right)-\left(\sum_{1 \leqslant i<j \leqslant n} \frac{4}{\left(x_{i}-x_{j}\right)^{2}}\right)
$$

where $x_{i}$ are the particle positions and $y_{i}$ are their momenta. This Hamiltonian bears a clear resemblance to the Calogero-Moser particle system $[1,8,11,15,16]$. In fact, it is similar to that famous system in several important ways.

Most importantly, we will observe that this system, like the Calogero-Moser, is integrable and self-dual. To see this, it is convenient to write $H$ in terms of the Calogero-Moser matrices $[10,16]$. That is, consider the set of all pairs of $n \times n$ matrices $(X, Z)$ satisfying

$$
\operatorname{rank}([X, Z]+I)=1 \text {. }
$$

An element of this set can naturally be associated with a state of the system in which the particles occupy distinct positions. In that case we consider

$$
X=x_{i} \delta_{i j} \quad Z=y_{i} \delta_{i j}+\frac{\sqrt{2}\left(1-\delta_{i j}\right)}{x_{i}-x_{j}} .
$$

(Note that as compared to the presentation of these matrices in other papers, a factor of $\sqrt{2}$ has been added to the matrix $Z$ off of the diagonal for later convenience.)

It was observed in [13] that, as in the case of Calogero-Moser, the Hamiltonian function can be written simply in terms of $X$ and $Z$ as follows:

$$
H=\operatorname{Tr}\left(Z^{2}-X\right) \text {. }
$$

Now observe that the map

$$
(X, Z) \mapsto(\bar{X}, \bar{Z})=\left(\left(Z^{\top}\right)^{2}-X^{\top}, Z^{\top}\right)
$$

is an involution on the space of matrices satisfying the rank one condition (2). More importantly, note that the corresponding Hamiltonian is

$$
\bar{H}_{j}=\operatorname{tr}\left((\bar{Z})^{2}-\bar{X}\right)^{j}=\operatorname{tr}\left(X^{j}\right)=\sum x_{i}^{j} .
$$

Since the Hamiltonian is independent of the $y$ values, the $x$ values are constant while the $y$ values change linearly. Since this map is a linearizing involution, we are thus able to conclude that $H$ is a self-dual integrable system.

Another way in which this system is similar to Calogero-Moser is that both systems describe the motion of poles in a solution to the KP hierarchy. In particular, as shown in [13], this Hamiltonian describes the dynamics of poles to a solution determined as an iterated Darboux transformation of the Airy solution [9]. Unlike Calogero-Moser, however, this solution is not a rational function of all of the time variables of the KP hierarchy and this solution does not correspond to a flow on the Jacobian variety of the spectral curve. In fact, the corresponding $\mathrm{KP}$ solution is associated with rank two bundles over the spectral curve, rather than rank one bundles as in the case of all particle systems for which the BQH has previously been tested. 


\subsection{Bispectrality and duality}

A linear differential operator $L$ in the variables $\vec{x}=\left\{x_{1}, \ldots, x_{n}\right\}$ is said to be trivially bispectral if there is a non-zero family of eigenfunctions $\psi(\vec{x}, \vec{z})$ parametrized by $\vec{z}=\left\{z_{1}, \ldots, z_{n}\right\}$ such that

$$
L \psi(\vec{x}, \vec{z})=p(\vec{z}) \psi(\vec{x}, \vec{z}) \quad \text { and } \quad \psi(\vec{x}, \vec{z})=\psi(\vec{z}, \vec{x}) .
$$

This is a special case of the more general bispectral property first considered in [4] which does not require that $\psi$ be symmetric, but only that it should also be an eigenfunction for an operator $\Lambda$ in $\vec{z}$ with eigenvalue depending on $\vec{x}$. (See [6] for a recent overview of this field and its diverse connections to mathematical physics.)

Bispectrality is related to the duality (cf $[7,14]$ ) of integrable particle systems both at the classical and the quantum levels. The main result of this paper concerns the quantum manifestation, but its role in the classical case is also relevant as motivation for the main result. Therefore, let us recall that the self-duality of the classical Calogero-Moser system was related to bispectrality in [8] and [16] where the linearizing map for this system was found to be equivalent to the exchange of spectral and spatial parameters in the KP wavefunction. In contrast, the self-duality of the higher rank classical systems presented above was conjectured in [9] due to the fact that they too describe the motion of the poles of bispectral KP Lax operators.

It is interesting that the bispectral problem led us to self-dual classical integrable systems because bispectrality is really the structure of quantum duality. That is, according to the $\mathrm{BQH}$ [5], dual systems when quantized should share an eigenfunction with spatial and spectral parameters reversed (and hence self-duality should be manifested as trivial bispectrality). A naive form of canonical quantization of the Hamiltonian function $H$ involves formally replacing $y_{i}$ with the differential operator $\partial_{i}$. This then leads us to the main question as follows:

Is there a non-zero eigenfunction $\tilde{\psi}$ satisfying the wave equation

$$
\tilde{\mathcal{H}} \tilde{\psi}(\vec{x}, \vec{z})=p(\vec{z}) \tilde{\psi}(\vec{x}, \vec{z})
$$

for some non-constant function $p$ and the operator $\tilde{\mathcal{H}}(1)$ with the symmetry

$$
\tilde{\psi}(\vec{x}, \vec{z})=\tilde{\psi}(\vec{z}, \vec{x}) ?
$$

\section{Intertwining relations}

The operators $L$ and $\tilde{L}$ are said to be intertwined by $K$ if they satisfy

$$
K L=\tilde{L} K \text {. }
$$

Such relationships are useful as transformations for producing an operator $\tilde{L}$ with specified spectral properties from a known $L$. For instance, this is one way to derive the quantum Calogero-Moser operator (the canonical quantization of the second Calogero-Moser Hamiltonian function) [3].

One begins with the constant-coefficient operator

$$
\Delta=\sum_{i=1}^{n} \partial_{i}^{2} \in \mathbb{C}\left[\partial_{1}, \ldots, \partial_{n}\right]
$$

in the commutative ring of constant-coefficient operators. Every element of this ring has the function

$$
\phi(\vec{x}, \vec{z})=\exp \left(x_{1} z_{1}+\cdots+x_{n} z_{n}\right)
$$

as an eigenfunction with eigenvalues depending on $\vec{z}$ and this is perhaps the most elementary example of trivial bispectrality. The following theorem of Chalykh and Veselov is provided here not only as an example but also as an important lemma. 
Theorem 3.1 ([3]). If there is a partial differential operator $\mathcal{D}_{n}(n \geqslant 2)$ which takes the form of a polynomial in $\partial_{i j}=\partial_{i}-\partial_{j}$ with coefficients rational in $x_{i j}=x_{i}-x_{j}(1 \leqslant i<j \leqslant n)$ such that

$$
\mathcal{D}_{n} \Delta=\tilde{\Delta} \mathcal{D}_{n} \quad \tilde{\Delta}=\Delta-\sum_{1 \leqslant i<j \leqslant n} 4 x_{i j}^{-2} .
$$

Then the function

$$
\tilde{\phi}(\vec{x}, \vec{z})=\prod_{1 \leqslant i<j \leqslant n}\left(z_{i}-z_{j}\right)^{-1} \mathcal{D}_{n}[\phi(\vec{x}, \vec{z})]
$$

is an eigenfunction for the Calogero-Moser Hamiltonian operator $\tilde{\Delta}$ satisfying $\tilde{\phi}(\vec{x}, \vec{z})=$ $\tilde{\phi}(\vec{z}, \vec{x})$.

It is an immediate consequence of this theorem that one can similarly construct the quantized Hamiltonian $\tilde{\mathcal{H}}$ via an intertwining relationship as detailed in the following theorem.

Theorem 3.2. Let

$$
\mathcal{H}=\sum_{i=1}^{n} \partial_{i}^{2}-x_{i} \quad \text { and } \quad \tilde{\mathcal{H}}=\mathcal{H}-\sum_{1 \leqslant i<j \leqslant n} 4\left(x_{i}-x_{j}\right)^{-2} .
$$

Then the operator $\mathcal{D}_{n}$ from theorem 3.1 satisfies

$$
\mathcal{D}_{n} \mathcal{H}=\tilde{\mathcal{H}} \mathcal{D}_{n} .
$$

Proof. Since $\left[\partial_{i}-\partial_{j}, x_{1}+x_{2}+\cdots+x_{n}\right]=0$, one has that

$$
\mathcal{D}_{n} \mathcal{H}=\mathcal{D}_{n}\left(\Delta-\left(x_{1}+\cdots+x_{n}\right)\right)=\left(\tilde{\Delta}-\left(x_{1}+\cdots+x_{n}\right)\right) \mathcal{D}_{n}=\tilde{\mathcal{H}} \mathcal{D}_{n}
$$

Remark. This is an interesting result to compare with the one-dimensional case. The ordinary differential operator $\partial^{2}$ and $\partial^{2}-2 / x^{2}$ are intertwined by the operator $\partial-1 / x$, which can be regarded as the one-dimensional case of theorem 3.1. In contrast, the Airy operator $\partial^{2}-x$ is unique among bispectral Schrödinger operators (cf [4]) in that it cannot be intertwined with another rational operator. Therefore, it may be seen as a somewhat surprising fact that $\mathcal{H}$, its higher-dimensional analogue, does intertwine with a rational operator.

\section{Eigenfunctions}

Still, this does not resolve the question of whether $\tilde{\mathcal{H}}$ has an eigenfunction which is symmetric in spatial and spectral parameters. Unfortunately, the first thing one might try turns out to be a 'wrong turn'. Since the operator $\mathcal{H}$ is contained in the commutative ring $\mathbb{C}\left[\partial_{1}^{2}-x_{1}, \ldots, \partial_{n}^{2}-x_{n}\right]$ (polynomials in $n$ different one-dimensional Airy operators) which has the symmetric common eigenfunction

$$
\sigma(\vec{x}, \vec{z})=\prod_{i=1}^{n} \operatorname{Ai}\left(x_{i}+z_{i}\right)
$$

one has immediately the following theorem.

Theorem 4.1. The operator $\tilde{\mathcal{H}}$ has eigenfunction $\tilde{\sigma}=\mathcal{D}_{n}[\sigma]$ satisfying the equation

$$
\tilde{\mathcal{H}} \tilde{\sigma}=\left(\sum z_{i}\right) \tilde{\sigma} \text {. }
$$


However, neither $\tilde{\sigma}$ nor any multiple of it by a non-zero function of $\vec{z}$ is symmetric in spatial and spectral parameters. This apparent counter-example to the $\mathrm{BQH}$ is resolved by recognizing that $\mathcal{H}$ is also contained in another commutative ring with another symmetric common eigenfunction.

Theorem 4.2. The operators $\partial_{i n}=\partial_{i}-\partial_{n}(1 \leqslant i<n)$ each commute with $\mathcal{H}$ and the commutative ring $\mathbb{C}\left[\partial_{1 n}, \ldots, \partial_{n-1 n}, \mathcal{H}\right]$ has the symmetric common eigenfunction

$$
\psi(\vec{x}, \vec{z})=\exp \left(\frac{1}{n} \sum_{1 \leqslant i<j \leqslant n} x_{i j} z_{i j}\right) \operatorname{Ai}\left(\left(\frac{1}{n}\right)^{1 / 3} \sum_{i=1}^{n}\left(x_{i}+z_{i}\right)\right)
$$

satisfying

$$
\partial_{i j} \psi=z_{i j} \psi=\left(z_{i}-z_{j}\right) \psi \quad \text { and } \quad \mathcal{H} \psi=p_{n}(\vec{z}) \psi
$$

for the polynomial

$$
p_{n}(\vec{z})=\sum_{j=1}^{n}\left(\left(\sum_{i=1}^{n} z_{i j}\right)^{2}+z_{j}\right)
$$

Proof. The easiest way to observe this is by direct computation. For instance, one may derive $p_{n}$ by first noting that

$$
\left(\partial_{j}^{2}-x_{j}\right) \psi=\left(\left(\sum z_{i j}\right)^{2}+\frac{1}{n} \sum\left(x_{i}+z_{i}\right)-x_{j}\right) \psi+\left(\sum z_{i j}\right) \psi^{\prime}
$$

for a function $\psi^{\prime}$ that does not matter since it disappears when one sums over $j$. However, a more instructive way to verify the claim is to consider the change of variables $\alpha_{i}=x_{i}-x_{n}$ $(1 \leqslant i<n)$ and $\alpha_{n}=x_{1}+x_{2}+\cdots+x_{n}$ after which the operator $\mathcal{H}$ decomposes into a sum of a constant-coefficient operator in $\alpha_{i}$ for $i<n$ and an Airy operator in $\alpha_{n}$.

Theorem 4.3. The function

$$
\tilde{\psi}(\vec{x}, \vec{z})=\prod_{i<j} z_{i j}^{-1} \mathcal{D}_{n}[\psi]
$$

satisfies the eigenvalue equation $\tilde{\mathcal{H}} \tilde{\psi}=p_{n}(\vec{z}) \tilde{\psi}$ and is symmetric (satisfying (4)).

Proof. Note that every differential operator $D(\vec{x}, \vec{\partial})$ acting on the function $\phi=\exp \left(\sum x_{i} z_{i}\right)$ merely acts as a multiplication operator, multiplying by the polynomial $D(\vec{x}, \vec{z})$ in $\vec{z}$ with coefficients that depend on $\vec{x}$. This is not necessarily true for differential operators acting on $\psi$, but it is true that a differential operator which can be written as a polynomial in $\partial_{i j}$ (as $\mathcal{D}_{n}$ can by construction) just multiplies $\psi$ by this same polynomial. In particular, $\tilde{\phi} / \phi=\tilde{\psi} / \psi=\mathcal{D}_{n}(\vec{x}, \vec{z})$ is the same polynomial in $\vec{z}$ and so the symmetry of $\tilde{\psi}$ is equivalent to the already verified symmetry of $\tilde{\phi}$.

\section{Conclusion}

We have seen that the self-duality of the quantum Hamiltonian $\tilde{\mathcal{H}}$ is directly related to the separability of the quantum Calogero-Moser Hamiltonian (specifically, the fact that $\tilde{\Delta}$ commutes with any operator in the variable $\left.\alpha_{n}=x_{1}+\cdots+x_{n}\right)$. This sheds light on the relationship between the system $\mathcal{H}$ and the Calogero-Moser system, and provides additional support for BQH.

Note that here $\tilde{\mathcal{H}}$ and $\tilde{\Delta}$ agree on the hyperplane $\sum_{i=1}^{n} x_{i}=0$ (which Chalykh-Veselov considered as a hypothesis anyway in order to avoid separability). Still, there remain essential 
differences between the two. In particular, one finds differences when considering the algebraic structure of the system since, in contrast to the Calogero-Moser system, there is no first-order operator commuting with $\tilde{\mathcal{H}}$.

The Hamiltonian system quantized above is only one example of many that were suggested by the results in [9]. See [13] for a hierarchy of classical Hamiltonian functions (of arbitrary order) which are linearized by an involution and hence should quantize bispectrally.

\section{Acknowledgments}

I am grateful for advice from and helpful conversations with $\mathrm{H}$ Braden, J Harnad, A Marshakov and J L Miramontes.

\section{References}

[1] Airault H, McKean H P and Moser J 1977 Rational and elliptic solutions of the Korteweg-DeVries equation and a related many-body problem Commun. Pure Appl. Math. 30 95-148

[2] Braden H W, Marshakov A, Mironov A and Morozov A 2000 Nucl. Phys. B 573 553-72

[3] Chalykh O A and Veselov A P 1990 Commutative rings of partial differential operators and Lie algebras Commun. Math. Phys. 125 597-611

[4] Duistermaat J J and Grünbaum F A 1986 Differential equations in the spectral parameter Commun. Math. Phys. 103 177-240

[5] Fock V, Gorsky A, Nekrasov N and Rubtsov V 2000 Duality in integrable systems and gauge theories J. High Energy Phys. Paper 2840

[6] Harnad J and Kasman A (ed) 1998 The Bispectral Problem (Providence, RI: Amercian Mathematical Society)

[7] Horozov E and Kasman A 1999 AMS/IP Studies Adv. Math. 13 289-97

Horozov E and Kasman A 1999 Lett. Math. Phys. 49 131-43

[8] Kasman A 1995 Commun. Math. Phys. 172 427-48

[9] Kasman A and Rothstein M 1997 Bispectral Darboux transformations: the generalized Airy case Physica D 102 159-73

[10] Kazhdan D, Kostant B and Sternberg S 1978 Hamiltonian group actions and dynamical systems of Calogero type Commun. Pure Appl. Math. 31 481-507

[11] Krichever I M 1978 Rational solutions of the Kadomtsev-Petviashvili equation and integrable systems of $N$ particles on a line Funct. Anal. Appl. 12 59-61

[12] Marshakov A 2000 Duality in integrable systems and generating functions for new Hamiltonians Phys. Lett. B $476420-6$

[13] Rothstein M 1998 The Bispectral Problem (Montreal, PQ, 1997) Am. Math. Soc. (Providence, RI: Amercian Mathematical Society) pp 105-10

[14] Ruijsenaars S N M 1988 Action-angle maps and scaterring theory for some finite-dimensional integrable systems Commun. Math. Phys. 115 127-65

[15] Shiota T 1994 Calogero-Moser hierarchy and KP hierarchy J. Math. Phys. 35 5844-9

[16] Wilson G 1998 Collisions of Calogero-Moser particles and an Adelic Grassmannian Invent. Math. 133 1-41 